\title{
Development and Practice of Project-based Curriculum in Higher Vocational Education by Using Simulation Technology
}

\author{
Xiaozhong Chen \\ School of information, Changzhou Vocational Institute of Engneering, Changzhou 213164, China. \\ chenxiaozhonghh@163.com
}

\begin{abstract}
In traditional skills training of computer network system integration, due to the lack of content, scale and application, and the lack of complete full network environment, the system integration capabilities of higher vocational students always can not be improved effectively. According to two goal posts in the computer network intergrated position group, namely, implementation engineer and management engineer, this paper proposed a neffective method to improve the practical ability of computer network professional students in campus network system integration, in which the core position competences were sumarized, and the supporting resources were developmented by course design based on simulation technology
\end{abstract}

Keywords: Computer network; simulation technology; post skill; project; curriculum development.

\section{Introduction}

In the past ten years, the reform of higher vocational education and construction of the curriculum has achieved fruitful results, the social recognition of highly skilled applied talents training was also significantly improved in China [1]. After the construction of demonstration school project in Jiangsu Province, Changzhou vocational institute, pays attention to professional connotation construction, the project-based curriculum teaching, and combining the concept into the teaching practice, with particular emphasis on the cultivation of students' vocational core competency. Under the background of the project-based curriculum teaching reform, the construction of computer network is regarded as a core course for the specialty of computer network technology. Over the past several years of exploration and practice, a set based on real projects and engineering specifications were formed. The experience and practice of simulation software in project-based curriculum development, especially students of post core skills training is quite remarkable.

\section{Locate Accurate Target Positions}

Based on the guiding principle of personnel training, services for economic and social development and employment guidance, in modern higher vocational education, the setting of professional curriculum core should be according to the specific goal posts, focusing on training students' professional skills. At present, the "Internet plus" has become the new norm of industrial development, the computer network system will be widely used in various industries and system oriented system integration post group also gradually subdivided into pre-sale, implementation, customer service, safety and management of network engineer. In a large number of research results[2] , the higher vocational computer network technology professional students were propsed to be more suitable for network integration network, implementation engineer and enterprises and institutions, network management engineer and other typical positions. Mastering the network planning, equipment commissioning and maintenance, network application management and other aspects of basic skills, are required for the two positions.

Teaching design of curriculum is one of the important things to improve training core skills. Computer network construction, as the core course of the computer network technology, was in the grasp of knowledge based on training typical job in campus network platform construction and application service deployment and integration ability. The teaching goal of the course is to master the working principle of switching, routing, security, equipment and management system; in accordance with the plan, construction foundation platform in planning, application service 
deployment in campus network, to meet with user's typical application requirements, planning and optimizing the network application; training project specification and implementation techniques and other content. In the end, the students can be competent for the design, deployment and maintenance of the integrated project of the medium scale network system.

\section{Curriculum Characteristics and Simulation Software}

\subsection{Curriculum Characteristics.}

With the promotion and implementation of teaching quality project in our country, the domestic vocational colleges were a large number of Internet equipment related courses teaching reform research and practice, also appeared a lot of project teaching achievement [3], however, is a single function of the local network configuration ability training project. Due to the limitation of the function and scale of the designed training program, the students are difficult to realize the integrated environment of many kinds of devices and applications in the whole network. Wiring technology, the server system configuration, professional courses, characterized by high for the integration of professional skills are integrated in the construction of computer network, many types and number of network equipment, application service environment require. In order to strengthen the training of students' practical skills, many domestic schools hesitate to invest a lot of money, various construction for the major of computer network training room, let students experience and skilled in the use of the manufacturers of the typical equipment, learning the solutions. Hardware laboratory construction, no doubt for the students of the individual skills training to provide a resource protection. However, even if the number of network hardware equipment, it is difficult to provide a complete network of teaching and training environment in the park, can not ensure that each student can independently control a network.

\subsection{Simulation Software.}

CISCO, HUAWEI, H3C and other well-known network equipment manufacturers, in the promotion of their products and qualifications of the respective companies, have launched a variety of computer network simulation software. Initially, Netsim Boson was developed primarily for experiments of CCNA and CCNP certification, but the function was limited to the certification of knowledge points. GNS3 was developed to read part of the IOS systems in the graphical interface. However, more emphasis was placed on a single technical level, lacking of systematic training programs designed from the teaching level of vocational education. With promotion of the Cisco Networking Academy Program in China, the advantage of the application of packet tracer has been widely supported. In China, there are also the packet tracer packages based on the supporting experimental tutorial, however, they are mostly discrete skills training experiment project, and the whole campus network the whole network simulation training project pen research has not yet found.

Simulation software, such as Tracer Packet, usually has the advantage of saving equipment investment and convenient use, which is regarded as the supplement of hardware experiment equipment. However, for computer network construction or network system integration and other course requiring of the higher integration, the advantages of simulation software is particularly prominent. First, the overhead of the system is very low, and a large number of different types of equipment, different application servers can be deployed. This makes it possible to build a network integrated training environment based on the application, and each student can be a typical network for a set of network equipment and application services. Secondly, the integration of the network environment, it can be convenient for students to carry out the study of unbounded learning at any time and at any site, to share the simulated resources, so that students can be widely used in skills training. Finally, based on the evaluation environment of simulation software, and it can quickly and comprehensively evaluate the students' ability of teaching and the students' self examination and teachers' curriculum evaluation. 


\section{Design of Project-based Course Aiming at Core Ability}

\subsection{Project-based Course Design.}

There have much in common in the core competence of two goal posts, engineers and network managers in the curriculum design. In this study, the core competence was summarized and extracted by the research related persons, including original equipment R \& D personnel, application service software developers, integrators network engineer, and enterprise network administrator. According to the necessary procedural knowledge and professional standards to determine the core competencies of the curriculum, involving the planning, construction and operation and maintenance services and other aspects of the campus network. The main goal of the course, computer network construction, is to cultivate students' integrated ability of network equipment and application services. Post-based core competence mainly includes the following steps: understanding IOS model and familiar with TCP/IP protocols; mastering switches, routers, firewalls, gateways, authentication and accounting equipment principle of work; skilled using VLAN, OSPF, STP, PPP and other agreement to build the foundation of network communication; mastering service system in DNS, DHCP deployment; to make corresponding changes to the project with the specific needs of the user; and quickly locating and troubleshooting network typical fault; to guarantee the project schedule and quality of construction, and complete all kinds of construction documents.

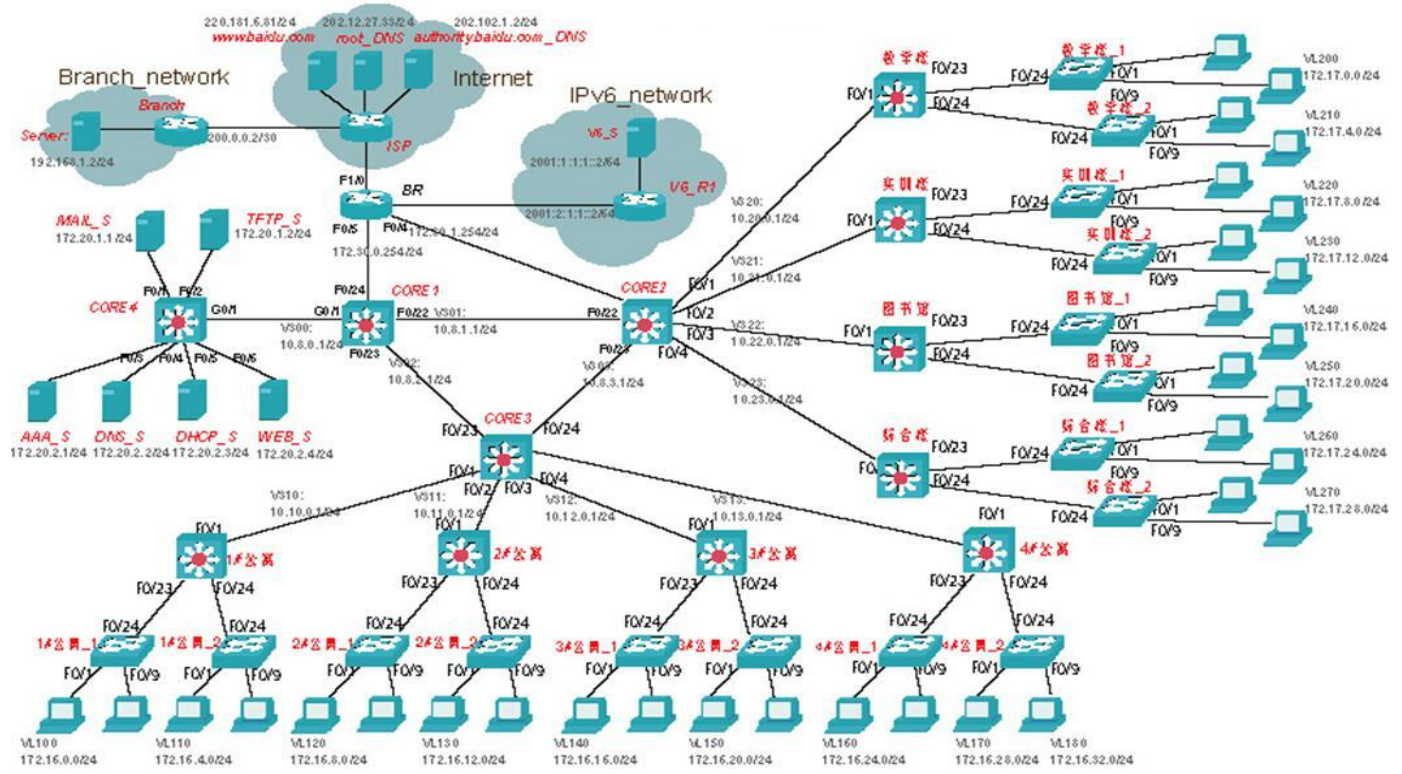

Fig. 1 Simulate Topology of Campus Network

Training post-based core competence through the typical project practice, the project curriculum should be designed to strengthen the professional training of students' action and operation training. Network systems integration projects due to the industry, the demand is different, the typical solution is different. Understanding the typical needs of the project and the actual environment, is an important prerequisite for the design and deployment of the network. Therefore, the author designed typical campus network structure as shown in Fig.1, so that students can have a relatively clear grasp of the project background and application requirements. The training content of the project-based course was designed according to the professional activities of the above posts. Constructed according to the network planning, network infrastructure, buildings interconnection, access control configuration, application service setup, export security deployment typical project and construction sectors, the campus network construction project was divided to a plurality of operation and inspection tasks. Each ability point one by one, link the students' professional skills. Progressive design of each sub task, can enhance the integration of the link skills points, and be easy to analyze, then locate and troubleshoot the faults.

\subsection{Learning Packages Design.}

Based on the post-based, the course training must be based on a typical project, in order to work process oriented and reflect the professional activities. Therefore, the development of project-based 
curriculum "learning packages" are particularly important for the course design of computer network construction. Taking into account the own advantages of Packet software, such as the combination of physical and logical model and the test function, the study resources packages (in Table 1) were developed from many views. On the one hand, development were based on the project construction process and standard design resources. The network equipment and link the physical connection, VLAN management, routing management, access control, access outside the network and application services etc. construction stage, resource development, were all taken into consideration. On the other hand, the combination of training documents and test documents. Pkt and PKA are the two file formats of packet tracer, the former supports basic configuration, which is an increase of test score function and the combination of both types of resources can facilitate students' autonomous training, examination, and can be conveniently stage or comprehensive test. In addition, the reference configuration file as a standard, can be used for students to imitate, learning engineering configuration of the specification and skills. As by editing the configuration file, in the command line, fast batch equipment configuration, rather than each input command, so as to improve project efficiency and experience in the implementation of the project of the engineering operation method.

Table 1 Types of Learning Packages

\begin{tabular}{ccc}
\hline Package Type & Explaination & Suffix Type \\
\hline Trainning document & Task \& Templates & doc \& pkt \\
Testing document & Stage \& Comprehensive Tests & pka \\
Reference document & Configuration scripts & txt \\
\hline
\end{tabular}

\section{Conclusion}

Integration practice of computer network system, as a result of covering a large number of different types of network equipment, network application service, existing hardware training methods are difficult to meet the large numbers of students independent control and management of the whole network, and they lead to the students in the classroom training content and network applications, there is a certain distance, unable to quickly adapt to the network system integration in the implementation of the engineer and engineer two jobs. Therefore, with the help of simulation software, according to the job responsibilities and standards, well-designed system integration project of curriculum, based on the typical process of construction project, the design and development of relevant supporting learning resources, the effective implementation of the full training is to enhance the professional ability in integration aspects of network system for vocational institute students.

\section{Acknowledgements}

This study was financially supported by the Foundation of Modern Educational Technology of Jiangsu Province [45382] and the Foundation of Changzhou Vocational Institute of Engineering [grant number [ZY6105016].

\section{References}

[1]. Yaping Wu. Summary of the Research on the Reform and Construction of Higher Vocational Education in Recent Ten Years [J]. Vocational Education Forum, (2011) (33).54-58.

[2]. Feiyue Hu, Wenda Deng, Chunrong Qiu. Computer Network Professional Talent Demand Level Segmentation and Professional Ability [J]. Computer Education. 2009 (5). 56-58.

[3]. Aiping Tan; Hanhui Hu; Jian Li. Practice of Teaching Reform of Network Interconnection Equipment in Higher Vocational Education [J].Vocational Education Research. 2010 (3).95-96. 